\title{
Micropower Design of an Energy Autonomous RF Tag for UWB Localization Applications
}

\author{
Davide Fabbri, Matteo Pizzotti, and Aldo Romani \\ Advanced Research Center on Electronic Systems \\ University of Bologna \\ Cesena, Italy \\ \{davide.fabbri21, matteo.pizzotti, aldo.romani\}@unibo.it
}

\begin{abstract}
This paper describes the architecture and the micropower design criteria of a battery-less, energy autonomous, individually addressable RF tag for UWB localization applications, with a focus on baseband circuitry. The tag includes a UHF rectifier, power conversion and management circuits, an addressable wake-up radio module, a microcontroller-based control unit, and circuits for UWB localization. The proposed circuit is suitable for UWB localization either by using passive backscattering of received UWB pulses, or by using active UWB pulses generators. Power for operation is scavenged from a modulated UHF carrier also used for addressing purposes. The circuit is implemented on discrete components in a $3.12 \mathrm{~cm}^{2} \mathrm{PCB}$ area. The circuit can wake-up from fully discharged states and operates at distances as high as $\mathbf{1 0 . 8} \mathrm{m}$ from a $2 \mathrm{~W}$-ERP source in the UHF 865-868 MHz RFID band with a $+1.8 \mathrm{dBi}$ receiving antenna. The quiescent power consumption of the tag is $3.88 \mu \mathrm{W}$, and the average power consumption at an addressing and activation rate of one time per second is $4.7 \mu \mathrm{W}$. The effectiveness of UWB localization was tested in a localization system based on time-difference-of-arrival (TDOA) estimations, consisting of multiple UWB readers and UHF transmitters.
\end{abstract}

Keywords-energy harvesting; localization; micropower circuits; power management; $R F I D ; U W B$; wake-up radio

\section{INTRODUCTION}

The emerging field of wireless sensor networks (WSNs) has been experiencing significant advancements in recent years. The purpose of such systems typically consists in monitoring and transmitting data related to sensed physical quantities. Moreover, a growing number of applications require awareness of the current position of mobile nodes with increasing levels of accuracy, as in logistics, elder healthcare, and navigation. Ultra-wide-band RF localization techniques hold the promise of fast and precise position measurements [1]. They are typically based on time-of-arrival (TOA) measurements performed by a synchronized array of external readers of known position. One reader collects all TOA information and calculates the time difference of arrival (TDOA) between tag and receivers. Hence, the position of the tag can be estimated. However, although successful applications are emerging [2], power consumption at node level is still high, which struggles with requirements in terms of pervasiveness, miniaturization and durations of tags.

The activities related to UWB localization have received founding by the European Space Agency (ESA) within the project "Localisation of Objects in Space through RF Tags (LOST)" under Grant ESA AO 1-8471/15/NL/LvH. The activities related to micro-power conversion have received funding from the ECSEL Joint Undertaking (JU) under grant agreement No 737434.

This JU receives support from the European Union's Horizon 2020 research and innovation programme and Germany, Slovakia, Netherlands, Spain, Italy. This work reflects only the authors' view and the JU is not responsible for any use that may be made of the information it contains.
Reductions in the power dissipated at node level might open to the development of energy autonomous solutions, in which the tag scavenges power for operation from RF signals [3] emitted on purpose. Backscatter communications and UWB localization [4] offer negligible power consumptions, at the cost of a reduction in communication range. Currently, stateof-the-art wirelessly powered CMOS circuits with localization capabilities [5] achieve operating ranges in the order of $10 \mathrm{~m}$. However, such tags consist of highly optimized CMOS microelectronic circuits involving high non-recurring engineering costs and low adaptability. Complementarily, this paper aims at demonstrating the feasibility of micropower energy autonomous RF tags with identification and localization capabilities based on an optimized design involving off-theshelf components. More specifically, it is shown how specific design optimizations grant performances similar to state-of-theart integrated tags. The use of standard components allows for cost effective and customizable solutions, still in a reasonably small footprint. The paper presents the architecture of a battery-less, energy autonomous, individually addressable RF tag with micropower consumption, and demonstrates its effectiveness for UWB localization applications.

\section{LOCALIZATION PRINCIPLE}

The scheme used to localize the tag is depicted in Fig. 1. The monitored area is equipped with one or more UHF sources used for power transfer and tag addressing, $\mathrm{N}_{\mathrm{rx}}$ reference

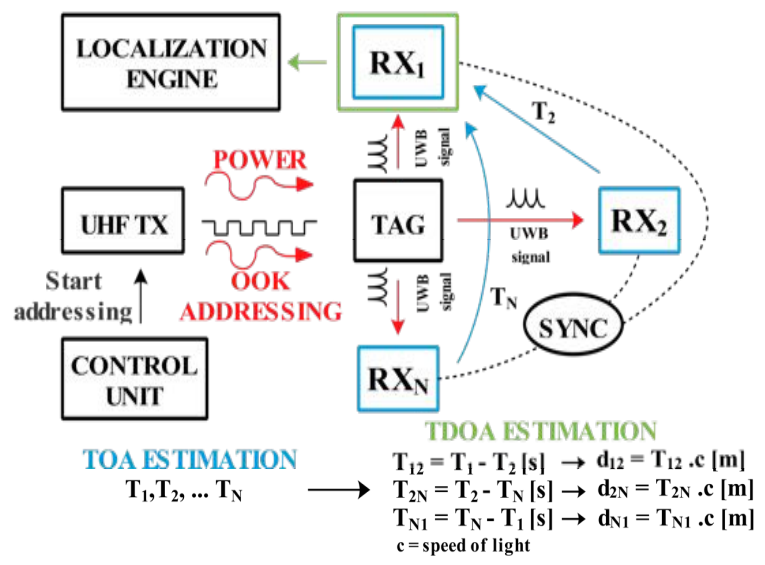

Fig. 1. TDOA localization scheme with reference nodes and RF powe transfer. 
nodes, a sync node, a control unit and the localization engine. Specifically, during the interrogation cycle performed by the Control Unit with a start operating signal, the tag is addressed by modulating the UHF carrier by using on-off keying (OOK). In between each interrogation, UHF sources transmit a continuous-wave for energy harvesting purposes. Once addressed, the tag transmits a pulsed UWB signal and returns into a sleep mode waiting for the next interrogation cycle. The reference nodes $\mathrm{RX}_{1}, \ldots, \mathrm{RX}_{\mathrm{N}}$ calculate the TOA between the tag and receivers and send their information to $\mathrm{RX}_{1}$, which estimates the TDOA. A sync node is deployed for time synchronization between readers, which improves localization accuracy. The localization engine combines all TDOA measurements and estimates tag position in space.

As a low-power alternative to active UWB transmission in tags, a UWB backscattering scheme can also be adopted [6], with the requirement of synchronizing tags and reference nodes. In this case, readers transmit the UWB signal, and TOA is measured on the backscattered signal.

\section{TAG ARCHITECTURE}

The architecture of the proposed micropower tag is shown in Fig. 2. The tag is compatible with UWB localization either (i) in a backscattering configuration [6] or (ii) with an active pulses generator [7]. The design is highly modular, so that individual modules are easily replaceable with more efficient parts as they become available. The tag is equipped with an UHF port, used for power harvesting and addressing, and an UWB RF port, used for localization purposes. It is worth mentioning that UHF and UWB antennas can be integrated in a single structure, as in [8]. Power incoming from the UHF port is rectified and converted into a DC form, which is managed by the on-board regulated DC/DC converter, which supplies tag circuitry when sufficient power is available and performs the maximum power point tracking (MPPT) of the RF source. The rectified UHF signal is also sent through a data slicer inspired by [9], which demodulates an on-off keying (OOK), actuated by the RF source on the UHF carrier during the addressing phase, and provides a digital signal. An ultra-low power microcontroller unit (MCU) is used for basic processing, for comparing the received bit stream with the locally stored individual tag address, and for generating control signals to drive either the backscattering switch or the pulses generation modules, according to the chosen tag configuration. A voltage monitor, integrated in the DC/DC converter, provides and cuts power to the MCU when the harvested energy is not sufficient to sustain an entire addressing phase and UWB transmission. Moreover, additional switches are used in both configurations to turn off external modules in order to reduce unnecessary quiescent power consumption.

\section{TAG OPERATION}

The heart of the tag is a low power MCU that manages the modes of operation of the entire circuit. In particular, the MCU starts in a LISTENING mode, in which it is running with a very-low-power low-frequency internal oscillator (VLO) and is waiting for the wake-up address to be sent by the UHF source. In this phase, the CPU is off and only the on-chip peripherals are clocked by the VLO in order to save power.

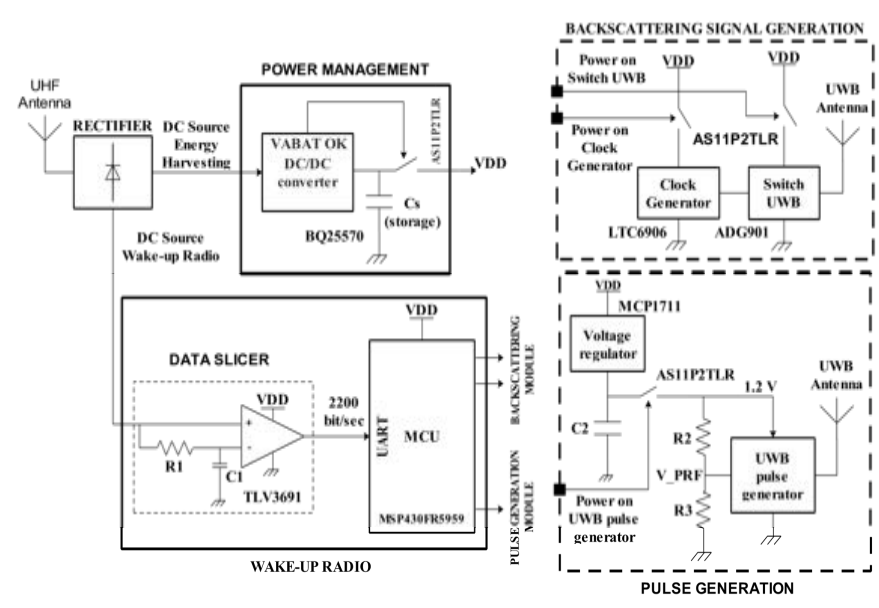

Fig. 2. Architecture of the proposed tag with the UWB transmission performed by using (i) the backscattering signal generation or (ii) pulses generation modules interchangeably.

The bit-stream received from the UHF antenna is filtered and converted to digital voltage levels by the data slicer circuit before being processed by the on-chip UART module of the MCU. When all the serial bits are received ( 8 data bits, 1 start bit and 1 stop bit with a data rate of $2200 \mathrm{bit} / \mathrm{sec})$, the CPU is woken up by an interrupt issued by the UART peripheral, and the address is software-decoded and compared with the tag address stored in the Flash memory of the MCU. The bits can also include redundancy for increased robustness. During this phase the mode of operation changes into ACTIVE mode and a higher-frequency internal clock (DCO) is enabled, which makes the MCU more responsive. Once the tag address is recognized, the microcontroller provides the signals used to power on the external UWB modules.

In particular, when considering the backscattering solution (Fig. 2) an ultra-low power external clock generator drives a RF switch in order to modulate the impedance of the UWB antenna (by alternating open-circuit and short-circuit configurations) and backscatter the received UWB pulses with a $0^{\circ}$ or $180^{\circ}$ phase shift. On the other hand, when considering active pulses generation with [10], the UWB pulses generator is supplied at $1.2 \mathrm{~V}$ by a linear regulator enabled by the MCU through an analog switch (Fig. 2). The voltage V_PRF, set with a resistive divider, modulates the UWB pulse repetition frequency. At this point, independently on the chosen configuration, after performing the basic actions for being localized, the tag needs to store energy for recharging a $22 \mu \mathrm{F}$ storage capacitor $\mathrm{C}_{\mathrm{S}}$. Each localization cycle consumes an amount of the energy stored in $\mathrm{C}_{\mathrm{S}}$ by the power management sub-system that needs to be replenished before subsequent activation. In case a different tag is addressed, a lower amount of energy will be consumed, mainly due to the only wake-up radio subsystem and to software address decoding.

\section{DESIGN TRADE-OFFS}

The best trade-offs with performance and low power consumption were pursued. The possibility to choose between different low-power modes and clock sources made the TI MSP430 microcontroller a reasonable choice for this work. In 
particular, during the addressing phase a slow data processing has been implemented. The MCU works in deep sleep LPM4 (Low Power Mode 4) which consumes only $400 \mathrm{nA}$ due to the VLO oscillator of $10 \mathrm{KHz}$ used to serve the UART peripheral. In this solution the very low power comparator TI TLV3691 with $75 \mathrm{nA}$ of quiescent current, suitable to process a slow data stream at few Kbit/sec, has been chosen. A complete address acquisition can be reached in $4.55 \mathrm{~ms}$ (with $2.2 \mathrm{Kbit} / \mathrm{sec}$ data rate). The comparison of the address bits is executed in ACTIVE mode at a frequency of $1 \mathrm{MHz}$ (DCO) in order to reduce the computation time. Concerning the application, this solution represents the best choice for very low power consumption. Where a higher data rate is required, in order to waste as little power as possible, a fast processing data can be chosen and all operation are done in high frequency by considering a comparator with lower propagation delay. Moreover, despite the relatively simple behaviour, a MCU offers greater flexibility with respect to custom logic and, most importantly, still a significantly lower energy consumption.

Considering the backscattering configuration, an external clock generator was used in order to have a higher accuracy in the generated frequency $(<0.2 \%)$ than with the internal DCO, which has a $1 \%$ of frequency clock drift. In addition, the LT LTC6906 oscillator has lower consumption and does not require external components. Ultra-low power crystal oscillators are not suitable because of their long start-up times of hundreds of ms, which compromise tag localization rate.

A technique for further reducing system power consumption consists in switching off all unnecessary devices during their idle time. Hence, it becomes convenient to use analog switches to turn off the external clock generator and UWB switch of the backscattering module, and the UWB pulse generator of the active module.

\section{EXPERIMENTAL RESULTS}

\section{A. Experimental setup}

The complete tag designed in a 4-layer PCB on a RF Rogers 4350B substrate is shown in Fig. 3. The tag was equipped with a single-monopole UHF (868 MHz) rectifying antenna derived from [11] with two output signals for addressing and for energy harvesting, respectively. The antenna gain is $1.8 \mathrm{dBi}$.

The quiescent power consumption of individual components was measured with a digital multimeter Agilent 34401A, and the results are reported in Table I.

TABLE I. QUIESCENT CURRENT CONSUMPTION OF COMPONENTS

\begin{tabular}{|c|c|c|}
\hline Component & Type & Current consumption \\
\hline bq25570 & DC/DC converter & $450 \mathrm{nA}$ \\
\hline AS11P2TLR & Analog switch & $100 \mathrm{nA}$ \\
\hline TLV3691 & comparator & $75 \mathrm{nA}$ \\
\hline MSP430FR5959 & microcontroller & $400 \mathrm{nA}$ \\
\hline MCP1711 & Voltage regulator & $600 \mathrm{nA}$ \\
\hline$[10]$ & UWB Pulse generator & $450 \mu \mathrm{A}$ \\
\hline LTC6906 & Clock generator & $12 \mu \mathrm{A}$ \\
\hline ADG901 & RF switch & $100 \mathrm{nA}$ \\
\hline
\end{tabular}

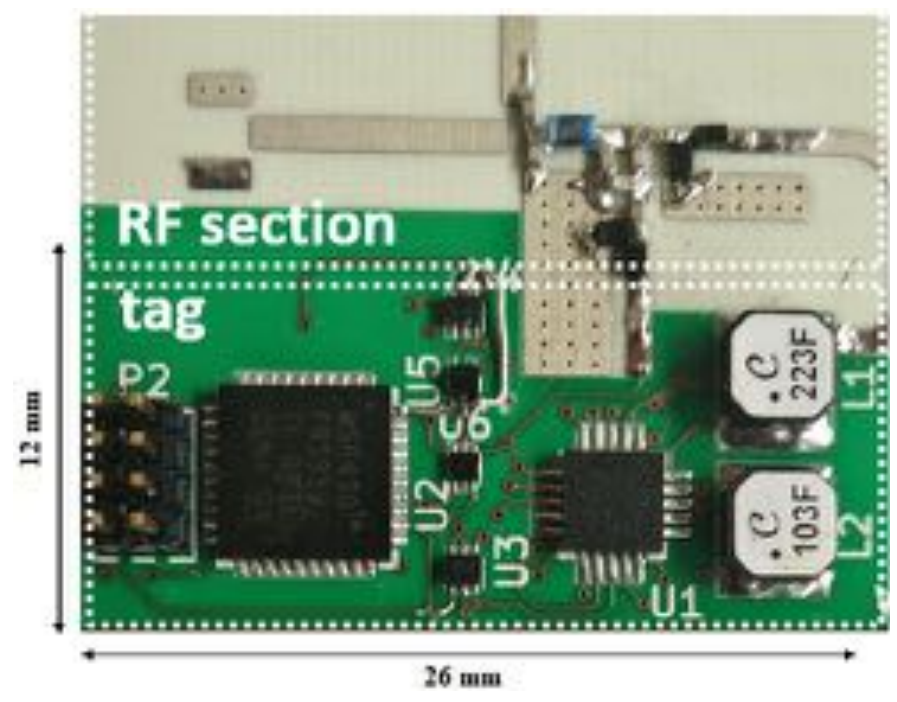

Fig. 3. PCB implementation of the baseband circuitry of the tag.

All the following results were obtained with the tag configured for active UWB pulses generation. The active tag was tested in an existing scenario depicted in Fig. 1, where 3 synchronized receiving nodes based on the Texas Instruments ADC12J4000 UWB RF sampling module estimate the TDOA of the UWB signal received from the tag after it is powered and addressed through a $2 \mathrm{~W}$-ERP UHF link. The number of used reference nodes allows a $2 \mathrm{D}$ localization process.

\section{B. Power consumption and maximum activation range}

The power and energy consumption of the tag, measured with the ST STEVAL-ISV021V1 power monitoring board, are reported in Fig. 4 and 5. In particular, the tag consumes 3.88 $\mu \mathrm{W}$ when waiting for an address to be sent. If we consider the whole sequence including also addressing and UWB pulse generation, the average power is $4.7 \mu \mathrm{W}$ at an activation rate of $1 \mathrm{~Hz}$. However, it is worth to mention that the power of the UWB signal transmitted by the custom pulse generator used in the tests is well below the allowed spectrum mask, which could limit localization range. We also stress that the development of the pulse generator is not part of this work and that the component was used to provide a more realistic test scenario.

Another experiment evaluated the maximum operating range of the tag. During power up, the high output resistance of

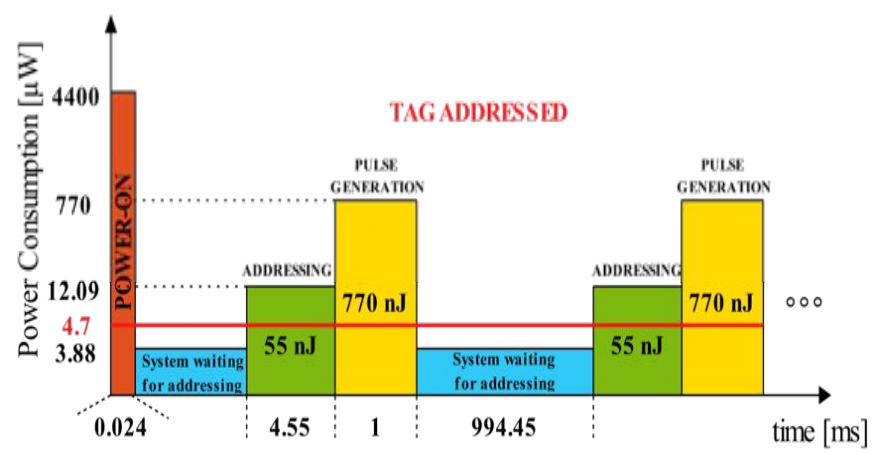

Fig. 4. Power and energy consumption of non-addressed tags at an activation rate of $1 \mathrm{~Hz}$. 


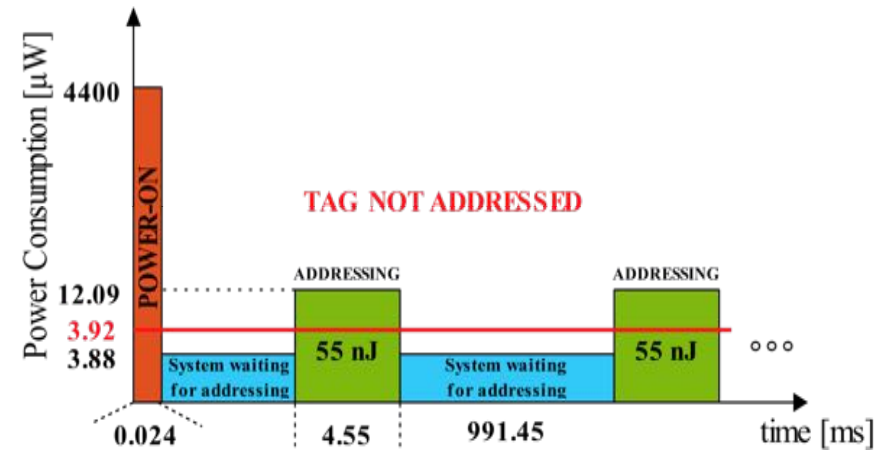

Fig. 5. Power and energy consumption of addressed tags at an activation rate of $1 \mathrm{~Hz}$.

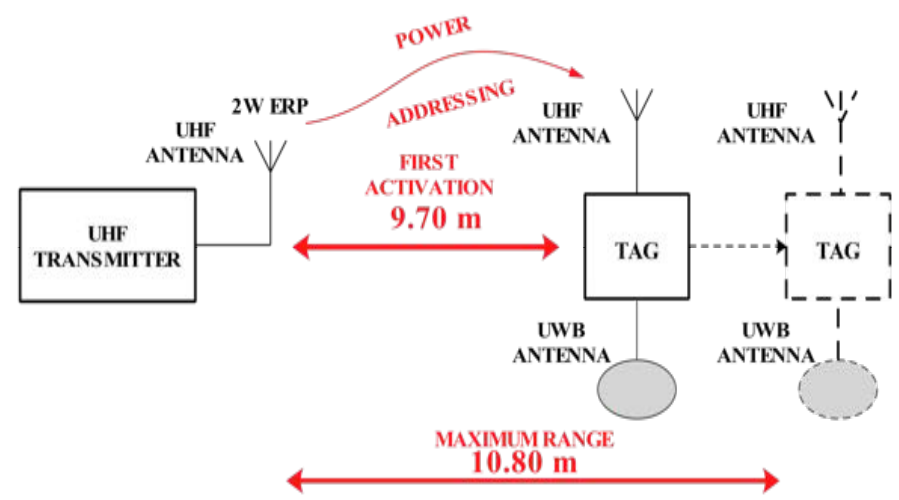

Fig. 6. Tag activation and maximum range distances.

the rectenna and the high in-rush current of the DC/DC converter causes significant voltage drops, which can prevent the $\mathrm{DC} / \mathrm{DC}$ converter from operating. The tag was brought in a fully discharged state at a high distance, and was slowly moved closer towards the UHF source until it was able to complete its power-up sequence. At this stage, the distance was increased again until the tag stopped operating.

As final result, the tag can be activated in few minutes from a fully discharge state, at a maximum distance from the UHF transmitter of $9.70 \mathrm{~m}$. In the same position, the performance ensures a maximum activation rate of $8 \mathrm{~Hz}$ and a missed activation rate of $7 \%$. Once the tag is turned on, it can be moved up to $10.80 \mathrm{~m}$, due to the lower power consumption after the start up.

\section{UWB localization}

The functionality of the tag was tested in the previously mentioned localization system with the indoor environment configuration shown in Fig. 7. In this case, the output of the pulses generator was connected to an externally powered amplifier, in order to compensate unexpected RF losses in the received samples. The amplifier restores the nominal output $R F$ power reported in [10]. So that the localization range becomes similar to the activation range. The position of tags was estimated by the localization system and compared with measurements performed with an optical precision laser system. The localization results of two different fixed position of the tag are reported in Table II, where the absolute error (AE) of the estimated position is in the best case $2 \mathrm{~cm}$. The obtained results are coherent with the expected performance of the existing localization system.

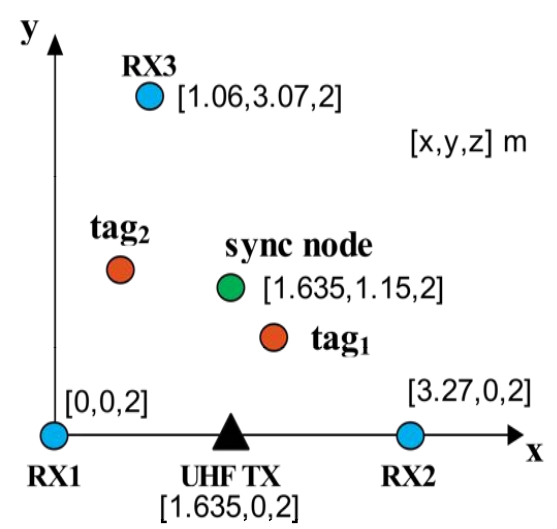

Fig. 7. Experimental set-up of the localization test.

TABLE II. LOCALIZATION RESULTS FOR TWO FIXED TAG POSITIONS.

\begin{tabular}{|l|c|c|c|}
\hline & $\begin{array}{c}\text { True position } \\
{[\mathbf{x}, \mathbf{y}, \mathbf{z}] \mathbf{( m )}}\end{array}$ & $\begin{array}{c}\text { Estimated position } \\
{[\mathbf{x}, \mathbf{y}, \mathbf{z}] \mathbf{( m )}}\end{array}$ & AE $[\mathbf{c m}]$ \\
\hline $\mathbf{t a g}_{\mathbf{1}}$ & {$[1.85,0.71,2]$} & {$[1.87,0.67,2]$} & 4 \\
\hline $\mathbf{t a g}_{\mathbf{2}}$ & {$[0.718,1.2,2]$} & {$[0.72,1.18,2]$} & 2 \\
\hline
\end{tabular}

\section{CONCLUSION}

A micropower RFID-UWB tag with wake-up radio capabilities and a flexible architecture has been implemented in a very small footprint with off-the-shelf components. The design is compatible with either UWB pulses generation or backscattering. Modularity allows to easily upgrade single parts with newer versions. Unless we make the UHF interface of the tag compliant with EPC Gen 2 specification as in [12], even a $0.5 \mathrm{~W}$ ERP transmitted power is still suitable to supply the tag, regarded that a more efficient DC/DC converter is used as in [13]. The use of micro-power components and an energy-aware design have extended maximum activation range to the distances achievable with UHF RFID technology. Hence, this work provides a suitable platform for battery-less applications that require accurate localization capabilities such as tracking of goods and people, logistics, as well as wirelessly-powered general-purpose sensor nodes.

\section{ACKNOWLEDGMENTS}

The authors thank Maxime Droguet, Christophe Craeye of the Catholic University of Louvain for setting up the localization system and for their support during the tests, and Nicolò Decarli, Anna Guerra, Davide Dardari of the University of Bologna for providing signal processing algorithms for localization and for their support during the experimental tests, and Marco Fantuzzi, Diego Masotti, Alessandra Costanzo of University of Bologna for designing the RF parts of the tag.

\section{REFERENCES}

[1] D. Dardari, A. Conti, U. Ferner, A. Giorgetti, and M. Z. Win, "Ranging With Ultrawide Bandwidth Signals in Multipath Environments," Proc. IEEE, vol. 97, no. 2, pp. 404 426, Feb. 2009.

[2] A. R. Jimenez Ruiz and F. Seco Granja, "Comparing Ubisense, BeSpoon, and DecaWave UWB Location Systems: Indoor Performance Analysis," IEEE Trans. Instrum. Meas., vol. 66, no. 8, pp. 2106-2117, Aug. 2017. 
[3] S. Gollakota, M. S. Reynolds, J. R. Smith, and D. J. Wetherall, "The Emergence of RF-Powered Computing," Computer (Long. Beach. Calif)., vol. 47, no. 1, pp. 32-39, Jan. 2014.

[4] N. Decarli, A. Guerra, F. Guidi, M. Chiani, D. Dardari, A. Costanzo, M. Fantuzzi, D. Masotti, S. Bartoletti, J. S. Dehkordi, A. Conti, A. Romani, M. Tartagni, R. Alesii, P. Di Marco, F. Santucci, L. Roselli, M. Virili, P. Savazzi, and M. Bozzi, "The GRETA architecture for energy efficient radio identification and localization," in 2015 International EURASIP Workshop on RFID Technology (EURFID), 2015, pp. 1-8.

[5] J. Kang, S. Rao, P. Chiang, and A. Natarajan, "Design and Optimization of Area-Constrained Wirelessly Powered CMOS UWB SoC for Localization Applications," IEEE Trans. Microw. Theory Tech., vol. 64, no. 4, pp. 1042-1054, Apr. 2016.

[6] F. Guidi, N. Decarli, D. Dardari, F. Natali, E. Savioli, and M. Bottazzi, "A Low Complexity Scheme for Passive UWB-RFID: Proof of Concept," IEEE Commun. Lett., vol. 20, no. 4, pp. 676-679, Apr. 2016.

[7] M. A. K. Jazairli and D. Flandre, "A 65 nm CMOS Ultra-Low-Power Impulse Radio-Ultra-Wideband Emitter for Short-Range Indoor Localization," J. Low Power Electron., vol. 11, no. 3, pp. 349-358, Sep. 2015.

[8] M. Fantuzzi, D. Masotti, and A. Costanzo, "A Novel Integrated UWBUHF One-Port Antenna for Localization and Energy Harvesting," IEEE Trans. Antennas Propag., vol. 63, no. 9, pp. 3839-3848, Sep. 2015.

[9] M. Magno, V. Jelicic, B. Srbinovski, V. Bilas, E. Popovici, and L. Benini, "Design, Implementation, and Performance Evaluation of a
Flexible Low-Latency Nanowatt Wake-Up Radio Receiver," IEEE Trans. Ind. Informatics, vol. 12, no. 2, pp. 633-644, Apr. 2016.

[10] M. A. K. Jazairli and D. Flandre, "A 65 nm CMOS Ultra-Low-Power Impulse Radio-Ultra-Wideband Emitter for Short-Range Indoor Localization," J. Low Power Electron., vol. 11, no. 3, pp. 349-358, Sep. 2015.

[11] M. Fantuzzi, M. Del Prete, D. Masotti, and A. Costanzo, "Quasiisotropic RF energy harvester for autonomous long distance IoT operations - IEEE Conference Publication," in 2017 IEEE MTT-S International Microwave Symposium (IMS), 2017, pp. 1345-1348.

[12] A. P. Sample, D. J. Yeager, P. S. Powledge, A. V. Mamishev, J. R Smith, "Design of and RFID-Based Battery-Free Programmable Sensing Platform", IEEE Transactions on Instrumentation and Measurement, vol. 57, no. 11, Nov. 2008.

[13] M. Pizzotti, L. Perilli, M. del Prete, D. Fabbri, R. Canegallo, M. Dini, D. Masotti, A. Costanzo, E. Franchi Scarselli, A. Romani, "A LongDistance RF-Powered Sensor Node with Adaptive Power Management for IoT Applications", Sensors, vol. 17, issue 8, 2017. 